\title{
Prediction of Aerosolized Fungal Spores by Determining Respiratory Intensity of the Growing Colony
}

\author{
Xian Li ${ }^{1}$, Tengfei (Tim) Zhang ${ }^{2,1^{*}}$, Jihong Wang ${ }^{1}$, Shugang Wang ${ }^{1}$ \\ ${ }^{1}$ School of Civil Engineering, Dalian University of Technology (DUT), Dalian 116024, China \\ ${ }^{2}$ Tianjin Key Laboratory of Indoor Air Environmental Quality Control, School of Environmental Science and Engineering, \\ Tianjin University, Tianjin 300072, China
}

\begin{abstract}
Aerosolization of fungal spores from moldy surfaces can cause adverse health effects. The spores of an older colony are more easily detached than those of a younger colony. When the growth age of a colony is known, the aerosolization of spores can be more accurately predicted. This study proposed the adoption of respiratory intensity as an indicator of the growth age of Aspergillus niger colonies. Respiratory intensity is the mass of carbon dioxide produced by the respiration of a colony per unit quantity of spores within a certain time. Two types of colonies, with homogeneous and heterogeneous age distributions, were aerosolized in a wind tunnel to determine the spore release proportion and validate the method. The results revealed that the respiratory intensity of the homogeneous colony is closely correlated with the growth age when the age of the colony is less than 14 days under standard growth conditions. The spore release proportion increases with the growth age and the blowing speed. The predicted spore release proportion for a heterogeneous colony is more precise when the colony is divided into multiple sub-colonies to characterize the growth age for each individual spore release proportion. The relative errors of the predicted spore release proportions as compared with the test values were less than $10 \%$.
\end{abstract}

Keywords: Respiratory intensity; Growth age; Fungal spore; Aerosolization; Release proportion.

\section{INTRODUCTION}

Approximately $21 \%$ of indoor air quality problems originate from microbial contamination (Meharzi et al., 2017; Humbal et al., 2018; Yao, 2018). Microorganisms include bacteria, viruses and fungi, among which fungi are a heterogeneous group that impact human health (Rim and Novoselac, 2009; Wu et al., 2016; Mu et al., 2019; Norback et al., 2019; Zhao et al., 2019). Fungal exposure contributes to allergies, inflammation and infection in human beings (Kallawicha et al., 2018; Park et al., 2018; Liu et al., 2019; Zhang et al., 2019). Routes of fungal exposure include respiratory inhalation and skin deposition once the fungal spores or fragments are aerosolized (Wang et al., 2018). Aerosolization is the process by which some physical substances are converted into particles that are small and light enough to be carried in the air (Milton et al., 2013; Wei et al., 2019).

When a fungal colony develops on a moldy surface, fungal propagules may be released from the surface into the

\footnotetext{
* Corresponding author.

Tel.: +86 41184706279 ; Fax: +86 41184706279

E-mail address: tzhang@dlut.edu.cn
}

indoor air (Gopalakrishnan et al., 2016). Fungal spores can easily be detached from the colonies by disturbance and then dispersed into the air (Frankel et al., 2014; Piekarska et al., 2018). For Aspergillus, the connection between two adjacent conidia in a spore chain is a septum (Gorny et al., 2001). The septum is fragile and easily broken by an external disturbance. If disturbance forces were greater than the binding forces of conidia with the colony, the spores detach. The spore size of Aspergillus (A.) niger ranges from 3.0 to $5.4 \mu \mathrm{m}$, and the density is close to that of water (Qi et al., 1997). When fungal spores are released into the air, there is a high likelihood of airborne transmission. Consequently, the concentration of fungal spores downstream from a colony can be meaningfully high and can result in indoor air pollution (Hugenholtz and Fuerst, 1992).

Aerosolization of fungal spores from moldy surfaces in indoor environments is driven mainly by aerodynamic forces (Wang et al., 2018; Fang et al., 2019). The dominating forces include drag and lift forces. The spore release rate from colonies was found to increase with the blowing air speed. Previous studies found that gusty airflow with high turbulence intensity could enhance spore aerosolization (Gopalakrishnan et al., 2016).

Experimental tests have been conducted to determine the aerosolization potential of fungal spores from colonies (Gorny et al., 2001; Kildeso et al., 2003). A piece of moldy 
material containing a fungal colony was placed in a source strength tester for measurement of the spore release rate (Sivasubramani et al., 2004). The measured values were then used to estimate the aerosolization potential of spores from other colonies. However, the above predicted spore concentration is not very accurate. One reason is that the aerosolization potential of the tested colony can differ greatly from that of other colonies. Growth status and age may vary greatly from colony to colony.

The growth age of fungal colonies has a significant effect on the spore detachment and aerosolization (Mensah-Attipoe et al., 2016; Teertstra et al., 2017). Generally, the detachment forces of spores for younger colonies are significantly greater than those for older colonies (Li et al., 2018). For an older colony, the connection between spores in a spore chain is generally weak. Hence, the spores of the older colony require smaller forces for detachment than the spores of a younger colony. In contrast, the younger colony has firm septa between spores. Consequently, the spores of the older colony are more easily aerosolized than those of the younger colony.

Apparently, if the growth age of a fungal colony is known, the spore aerosolization can be more accurately predicted. However, the growth age cannot be determined directly from the appearance of the colony. An indicator is needed to characterize the growth age. The length of the mycelium has been used to indicate the growth rate of a colony and thus the growth age (Gervais et al., 1999), but this length does not continue to increase after the colony has grown for dozens of hours. Other potential indicators are the amounts of glycerol and mannitol in spores, which were found to differ significantly among colonies of different growth ages (Teertstra et al., 2017). Unfortunately, the variations in these amounts with the growth age were non-monotonic.

Meanwhile, respiratory intensity is an indicator that represents the metabolism of a fungal colony (Willcock and Magan, 2000). In the field of physiology, respiration is the movement of oxygen from the outside environment to the cells within tissues, and the transport of carbon dioxide occurs in the opposite direction (Atkin et al., 2010). Previous study adopted respiratory intensity as an indicator to identify strong or weak respiration of an organism (Ryan and Law,
2005). Respiratory intensity is the mass of carbon dioxide released by an organism per unit area or per unit quantity of cells within a certain time. In general, the respiratory intensity increases with the metabolism of the fungal colony. Because the metabolism of a colony varies dynamically with the growth age, the respiratory intensity may be correlated with the growth age of the fungal colony.

Until the present time, none of the existing studies has reported determination of the growth age of a colony from a physical quantity that is easy to measure. The aim of this study was to adopt respiratory intensity as an indicator to characterize the growth age of $A$. niger colonies for prediction of the spore aerosolization. The relationship between the spore release proportion and the growth age of the $A$. niger colonies under different blowing air speeds was established. Two types of colonies, with homogeneous and heterogeneous spore age distributions, were aerosolized in a wind tunnel to count the spore release proportion and validate the method.

\section{METHODS}

In this section, the basic principles for measuring the respiratory intensity of fungal colonies are presented first, followed by aerosolization tests of fungal spores in a wind tunnel. The strategy for predicting spore aerosolization for an unknown colony is addressed.

\section{Respiratory Intensity Tests}

In this study, respiratory intensity was defined as the mass of carbon dioxide released by the respiration of the fungal colony within a certain time per unit quantity of spores. Because a certain quantity of spores was produced per conidiophore of the colony under the standard growth conditions (Krijgsheld et al., 2013), the biomass of the colony was represented by the quantity of spores. The respiratory intensity was measured using the chemical absorbent method in a static chamber (Ryan and Law, 2005). For quantification of the released carbon dioxide, it was absorbed by a sodium hydroxide standard solution in preparation for acid-base titration. In a given test, as shown in Fig. 1, a patch of $A$. niger colony was first placed onto a holder inside a jar, containing

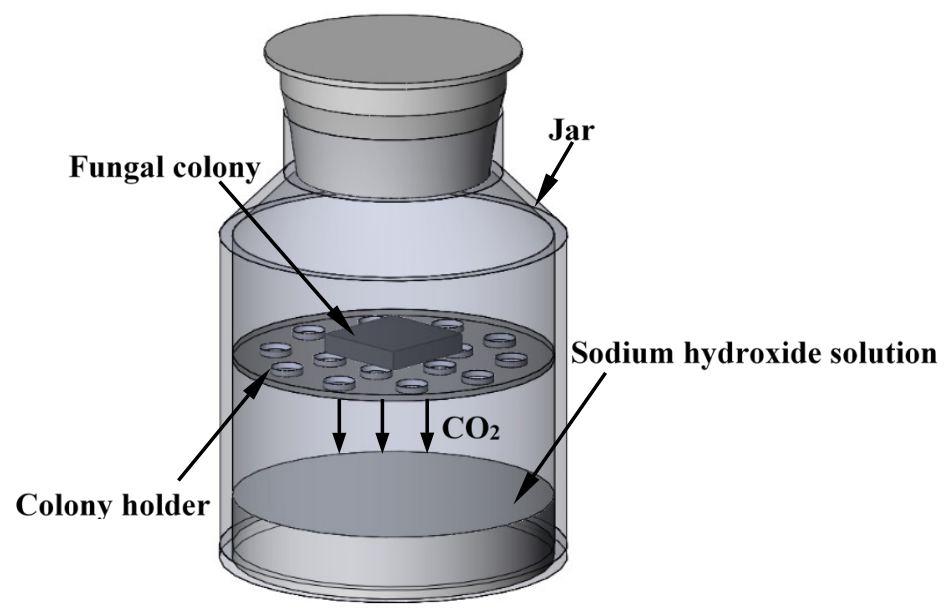

Fig. 1. Schematic diagram of measurement of respiratory intensity of a fungal colony. 
a certain amount of sodium hydroxide standard solution. The jar was then sealed and placed into an incubator for a certain duration. The fungal colony therein respired continuously and produced carbon dioxide, which was absorbed by the sodium hydroxide standard solution below the colony.

Next, the acid-base titration was carried out to determine the amount of carbon dioxide released by respiration. An acid-base titration is a method to determine the concentration of an acid or alkali by exactly neutralizing it with a standard solution of alkali or acid with the known concentration. The test colony was removed from the jar, and several drops of phenolphthalein indicator were added to the sodium hydroxide solution. The color of the solution changed from colorless to bright red. The solution was then titrated with hydrochloric acid standard solution until the phenolphthalein indicator became colorless. The consumption of sodium hydroxide solution by the respiration of the fungal colony was then determined.

Sodium hydroxide solution can also absorb carbon dioxide from the surrounding air. To quantify the absorbed carbon dioxide from the background air, we replaced the $A$. niger colony with a blank agar plate without spores on it. The consumption of sodium hydroxide solution by fungal respiration was the consumption of the sodium hydroxide solution for the test of the $A$. niger colony subtracted by that for the test of the blank agar plate.

The dimensions of the test colony were $0.5 \mathrm{~cm} \times 0.5 \mathrm{~cm}$; this was the minimum size for measuring the respiratory intensity because of the limited precision of the measuring instrument. We kept the jar with the colony in the incubator for 24 hours when measuring the released carbon dioxide. The temperature inside the incubator was $28^{\circ} \mathrm{C}$ with a relative humidity (RH) of $60-65 \%$, and the same conditions were used for incubating the test samples. The volume of the sodium hydroxide standard solution in the jar was $20 \mathrm{~mL}$ with a concentration of $0.100 \mathrm{~mol} \mathrm{~L}^{-1}$. The concentration of the hydrochloric acid standard solution was also $0.100 \mathrm{~mol} \mathrm{~L}^{-1}$. The consumption of sodium hydroxide standard solution per $\mathrm{mL}$ with $0.100 \mathrm{~mol} \mathrm{~L}^{-1}$ is equivalent to the release of $2.2 \mathrm{mg}$ carbon dioxide by the respiration of the colony. Five repeated measurements were performed for each test sample.
Determination of respiratory intensity also requires the grown spore density of the fungal colony. A parallel patch of $A$. niger colony was placed in a sterile culture dish, and the spores were scraped from the colony using an inoculating loop. Spore suspensions were made by washing the spores with a certain amount of sterile water. The concentration of the spore suspension was measured using a Bright-Line hemocytometer (Type 3900, Hausser Scientific, USA), and the spore density of the fungal colony was then determined.

Respiratory intensities of the colonies at different growth ages were measured. Finally, the variation in respiratory intensity with growth age was established.

\section{Aerosolization Tests}

The growth age of the fungal colony has a significant effect on spore detachment and aerosolization. For prediction of spore aerosolization, the spore release proportion for the colony at different growth ages was determined. The $A$. niger colonies at different growth ages were aerosolized in a wind tunnel for determination of the spore release proportion.

A simplified wind tunnel, as shown in Fig. 2, was used for the aerosolization test, just as we did previously (Li et $a l ., 2019)$. The dimensions of the test section were $150 \mathrm{~cm}$ $\times 30 \mathrm{~cm} \times 30 \mathrm{~cm}$, and the exit of the contraction section was smaller with dimensions of $15 \mathrm{~cm} \times 15 \mathrm{~cm}$. A patch of fungal colony with an area of $2 \mathrm{~cm} \times 10 \mathrm{~cm}$ was placed on the holder in the front of the test section. This area of the fungal colony was chosen to ensure that the aerosolized spores could be collected and detected. Aerosolized spores were extracted using a sharp-edged sampler and then counted by a wideband integrated bioaerosol sensor (WIBS) (Type NEO; DMT, USA). The sample flow rate of the WIBS was $0.3 \mathrm{~L} \mathrm{~min}^{-1}$. The inner diameter of the sampling tube was $0.8 \mathrm{~mm}$. Because the sample flow rate of the WIBS was much lower than the airflow rate in the wind tunnel, only a small quantity of aerosolized spores was detected by the sensor.

The spore release proportion from the colonies was defined as the ratio of the quantity of aerosolized spores to the total quantity of spores in the test colony before the aerosolization test. The quantity of aerosolized spores was estimated from the sampled spore concentration and the

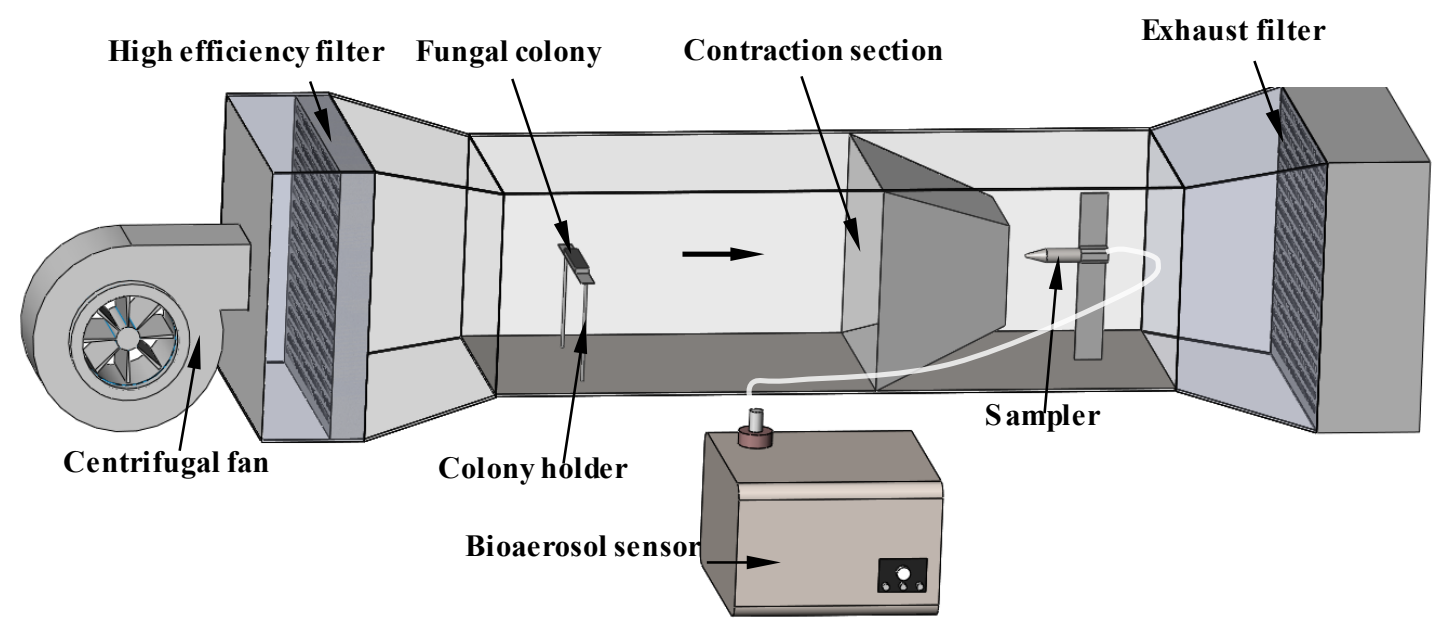

Fig. 2. Schematic diagram of a wind tunnel for aerosolization of spores from a fungal colony. 
wind tunnel airflow rate. To test the uniformity of the airborne spores at the exit of the contraction section, the sampler collected airborne spores at nine equidistant sampling positions under the same test conditions. Each aerosolization test for a single sampling position required a new parallel colony. Consequently, many identical parallel colonies were required for the tests. The difference in the quantity of collected spores between the nine sampling positions was less than $10 \%$. The volumetric rate in the wind tunnel was measured by an air capture hood (Type 8380; TSI, USA), with a resolution of $1 \mathrm{~m}^{3} \mathrm{~h}^{-1}$ and an accuracy of $\pm 3 \%$ of reading $\pm 12 \mathrm{~m}^{3} \mathrm{~h}^{-1}$.

Prior to each aerosolization test, the wind tunnel was disinfected with $70 \%$ ethanol and then purified with cleaned air. The duration of each test was five minutes. The blowing speeds in the test section ranged from 1 to $6 \mathrm{~m} \mathrm{~s}^{-1}$. The temperature and $\mathrm{RH}$ ranges inside the test section were 15 $17^{\circ} \mathrm{C}$ and $40 \%-45 \%$, respectively. Each aerosolization test was repeated five times. After aerosolization tests of the colony at different growth ages, the relationship between the spore release proportion and the growth age under different blowing air speeds was established.

\section{Strategy to Predict Spore Aerosolization}

In this study, two types of colonies, with homogeneous and heterogeneous age distributions, were adopted as test samples. The colony with a homogeneous age (Fig. S1) was used to establish the relationship between spore release proportion and growth age. For the homogeneous colony, the spores were produced nearly simultaneously, and the growth age of the colony was homogeneous. However, most realistic colonies can have heterogeneous ages. In the example colony in Fig. S2, the age in the center was the oldest, while the age at the outer edge was the youngest, because the colony grew from the center outward and formed a heterogeneous age distribution.

Two methods were used to predict the spore release proportion for the unknown colony, i.e., the global method and the partition method. In the global prediction method, the spore release proportion was estimated from the average age of the whole colony according to the above established relationship between spore release proportion and growth age. To determine the average age, the respiratory intensity of the whole colony was measured, and then the average age was estimated from the respiratory intensity. In principle, the global prediction method was suitable for the homogeneous colony, while it might provide an estimated average age for the heterogeneous colony as a whole.

In the partition prediction method, the colony was divided into a number of sub-colonies, each of which had its own age. The spore release proportion for each sub-colony was estimated from the above established relationship between the release proportion and the growth age. The spore release proportion for the whole colony can be summed up as:

$$
P=\frac{\sum_{i=1}^{n} P_{i} N_{i}}{N}
$$

where $P$ is the spore release proportion for the whole colony, $P_{i}$ is the spore release proportion for the number $i$ sub-colony $(1 \leq i \leq n), n$ is the quantity of sub-colonies, $N_{i}$ is the quantity of spores in the number $i$ sub-colony, and $N$ is the total quantity of spores in the whole colony. In most cases, the partition prediction method would be required for prediction of spore release for a heterogeneous colony.

Fig. 3 shows the entire flow chart for predicting the spore release proportion for an unknown colony. There were four steps. First, the respiratory intensity of the homogeneous colony at different growth ages was measured, and the variation in respiratory intensity with growth age was established. Second, aerosolization tests of spores for the homogeneous colony at different growth ages under different blowing air speeds were conducted in a wind tunnel. The spore release proportion of the colony at specific growth ages and blowing air speeds was then determined. Third, for the unknown colony, the respiratory intensity of the whole colony was measured to determine the average age, or the whole colony was divided into sub-colonies to determine the age distribution by measuring their individual respiratory intensities. The spore release proportion for the whole colony was then estimated from the relationships established in the first and second steps. Fourth, the predicted spore release proportion for the unknown colony was validated by the aerosolization tests.

For $A$. niger colonies grown under the same conditions as in our investigation, a researcher might need to conduct only Step 3 to predict the aerosolized $A$. niger spores. However, for other fungi or for $A$. niger colonies grown under different conditions, Steps 1 through 4 must be implemented to establish the relationship between respiratory intensity and growth age and the relationship between growth age and spore release proportion. In addition, validation of the accuracy in the above two relationships is necessary.

\section{RESULTS AND DISCUSSION}

\section{Respiratory Intensities of Homogeneous Colonies of A. niger at Different Growth Ages}

After test samples of the homogeneous colonies of A. niger had been prepared under the standard growth conditions of $28^{\circ} \mathrm{C}$ and a humidity close to saturation, Step 1 in the flow chart in Fig. 3 was conducted. Details of the test microorganisms are provided in Supplementary material.

Fig. 4 illustrates the relationship between respiratory intensity and growth age for the homogeneous colony of A. niger. The respiratory intensity decreased with the growth age when the growth age was less than 14 days. However, the respiratory intensity profile tended to be flat and was independent of the age, if the growth age exceeded 14 days. The youngest measured colony was two days old, and it had a maximal respiratory intensity of $8,781 \mu \mathrm{g}\left(10^{7} \text { spores }\right)^{-1}$. Referring to the evolution of an A. niger colony, as shown in Fig. S3, the two-day-old colony was in the period of spore generation and had a vigorous metabolism. After two days, spores were produced gradually, and the mycelial structure tended to be complete. The metabolism of the colony decreased, and the respiratory intensity dropped accordingly. 


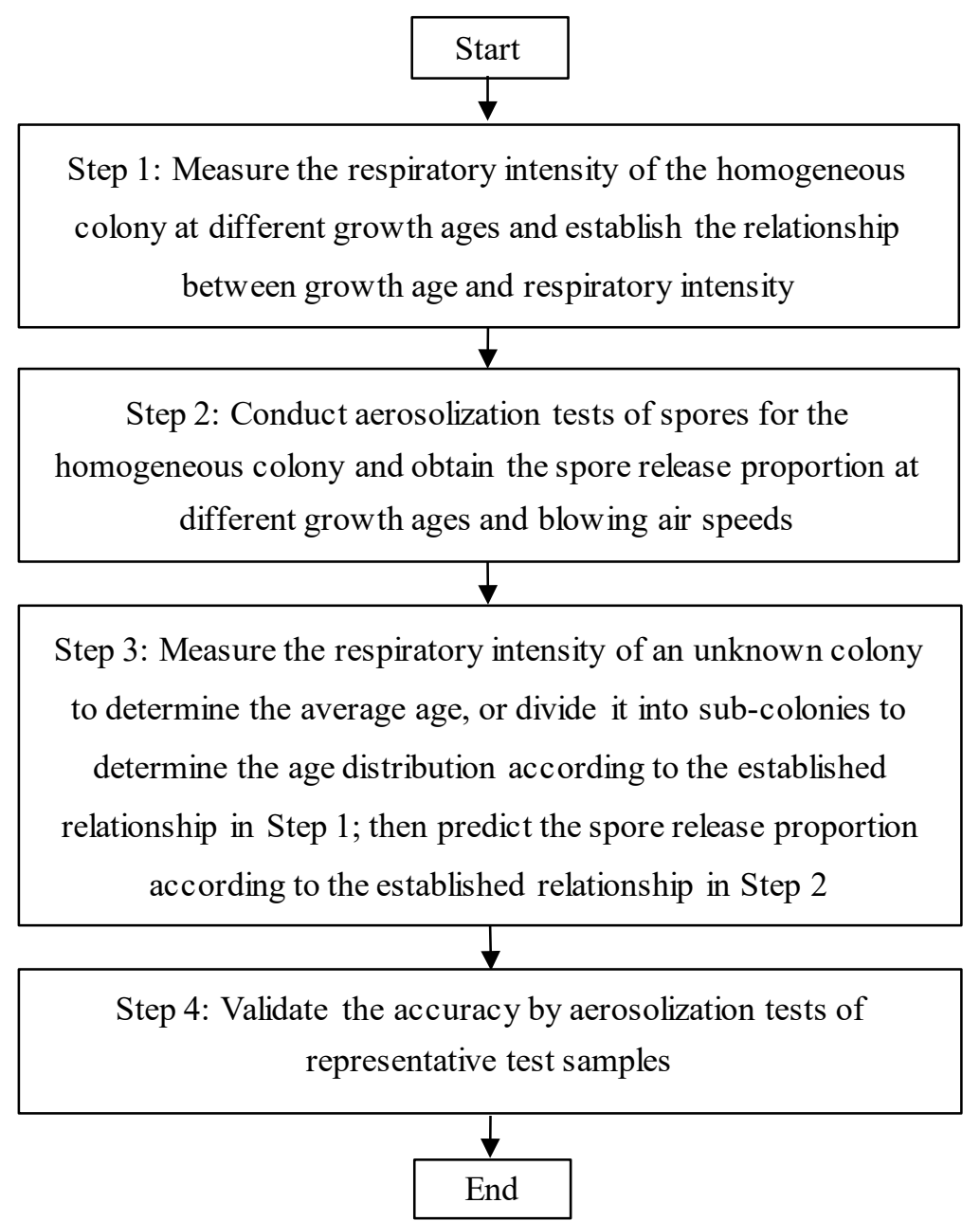

Fig. 3. Flow chart to predict the spore release proportion for an unknown colony.

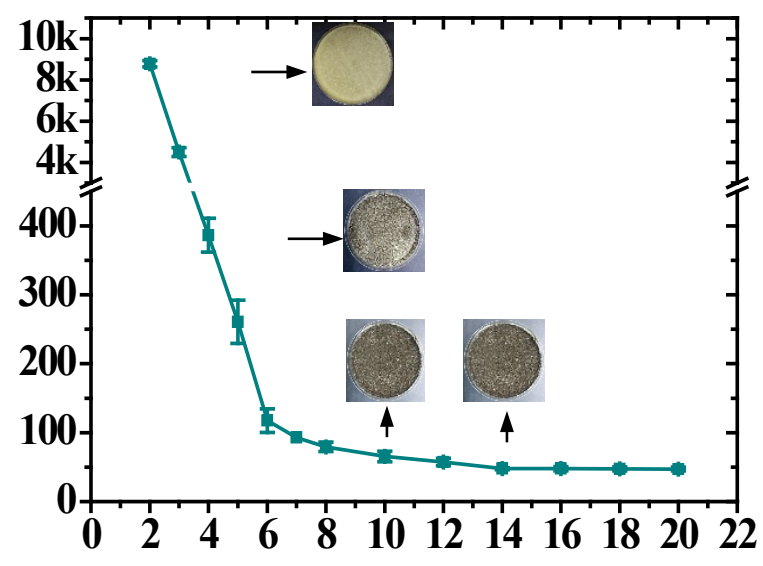

Fig. 4. Relationship between respiratory intensity and growth age for the homogeneous colony of $A$. niger, where error bars represent the standard deviations for five repeated measurements.

The respiratory intensity of the four-day-old colony had decreased sharply to $387 \mu \mathrm{g}\left(10^{7} \text { spores }\right)^{-1}$. As the growth age increased beyond six days, however, the decrease in respiratory intensity was gradual. The respiratory intensity of the eight-day-old colony was $79 \mu \mathrm{g}\left(10^{7} \text { spores }\right)^{-1}$, while the value for the 10 -day-old colony was $65 \mu \mathrm{g}\left(10^{7} \text { spores }\right)^{-1}$. The respiratory intensity reached its minimum $(47 \mu \mathrm{g}$ $\left.\left(10^{7} \text { spores }\right)^{-1}\right)$ when the growth age exceeded 14 days. The results indicate that the respiratory intensity is closely correlated with the growth age when the growth age is less than 14 days. Thus, for an unknown colony, the age can be determined from the respiratory intensity of the colony according to the above established relationship between respiratory intensity and growth age.

For the measurements of respiratory intensity, the amount of carbon dioxide released by the respiration of the fungal colony was determined by acid-base titration. The resolution of the burette was $0.01 \mathrm{~mL}$, which corresponded to approximately $0.02 \mathrm{mg}$ of carbon dioxide. Because of the limited resolution of the test instrument, small differences in respiratory intensity when the growth age exceeded 14 days could not be distinguished. A higher resolution instrument is needed to measure the respiratory intensity of colonies older than 14 days. In addition, the respiratory intensity may tend to be stable when the colony is sufficiently old. Other physical or chemical indicators are needed to characterize the growth age of the colony beyond 14 days. 
The respiratory intensity was determined as the mass of carbon dioxide released by the respiration of the fungal colony in 24 hours per a unit quantity of spores. The measurement duration is not meaningless, because the test colony might produce new spores that release carbon dioxide within the 24 hours of measurement. This investigation did not consider the newly produced spores when reporting the respiratory intensities. Further effort is required to measure the respiratory intensity of a fungal colony within a shorter duration.

\section{Spore Release Proportion for the Homogeneous Colonies at Different Growth Ages}

In accordance with Step 2 in the flow chart, the spores of colonies at different growth ages were aerosolized in a wind tunnel. Fig. 5 presents the spore release proportion for the homogeneous colonies at growth ages from four to 20 days subjected to a blowing air speed of $3.00 \mathrm{~m} \mathrm{~s}^{-1}$. Because the release proportion of the spores for the two-day-old colony was quite small, beyond the detection threshold of the bioaerosol sensor, the release proportion for the two-day-old colony is not presented. The spore release proportion for the eight-day-old colony was $0.93 \%$, which was 3.2 times that for the six-day-old colony $(0.29 \%)$. At a growth age of 14 days, the spore release proportion was $1.16 \%$. The release proportion of spores increased slightly with the growth age once the age was greater than 14 days. The results indicate that the spore release proportion increases with the growth age of the colony, while the respiratory intensity decreases with the growth age (Fig. 4). Although the variation trends for the two physical indicators with growth age are opposite, both the spore release proportion and respiratory intensity are significantly affected by the growth age when the age is less than 14 days.

Fig. 6 shows the spore release proportion for the homogeneous colonies versus the blowing air speed at different growth ages. The spore release proportion increased with the air speed. For the four-day-old colony, the release proportion under the blowing air speed of $3.50 \mathrm{~m} \mathrm{~s}^{-1}$ was $0.73 \%$, which was approximately 2.1 times that under the blowing speed of $3.25 \mathrm{~m} \mathrm{~s}^{-1}(0.35 \%)$. Hence, the blowing air speed of

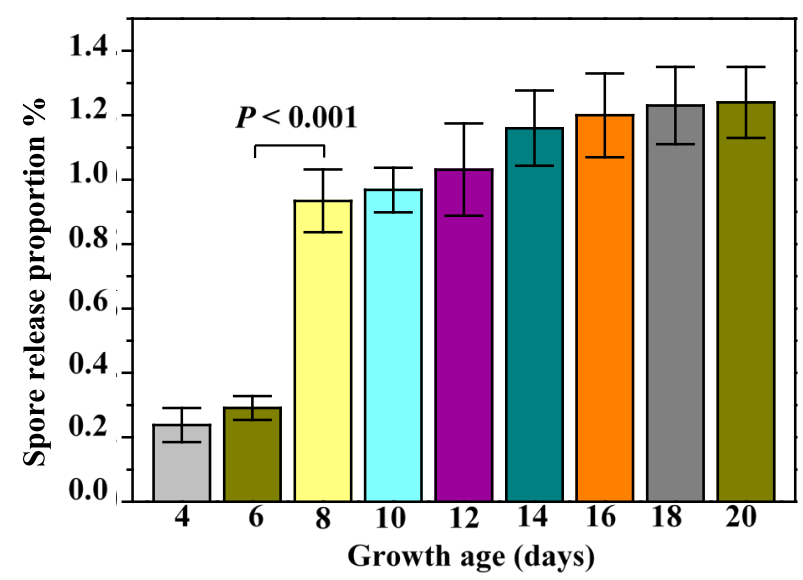

Fig. 5. Comparison of the spore release proportion for homogeneous colonies at different growth ages under a blowing air speed of $3.00 \mathrm{~m} \mathrm{~s}^{-1}$, where error bars represent the standard deviations for five repeated measurements.
$3.50 \mathrm{~m} \mathrm{~s}^{-1}$ was the threshold speed for significant detachment of spores from the four-day-old colony. For the six-day-old colony, the spore release proportion under the blowing speed of $3.25 \mathrm{~m} \mathrm{~s}^{-1}$ was approximately 2.8 times that under the blowing speed of $3.00 \mathrm{~m} \mathrm{~s}^{-1}$. Thus, $3.25 \mathrm{~m} \mathrm{~s}^{-1}$ was the threshold speed for significant detachment of spores from the six-dayold colony. The threshold blowing speeds for significant detachment of spores, as indicated by the red dots and corresponding speed values in the figures, decreased with the growth age. The spore release proportions were nearly identical once the growth ages exceeded 14 days.

For the determination of spore release proportion, the quantity of aerosolized spores was estimated from the number of detected spores. The average air speed in the sampling tube connecting to the bioaerosol sensor was approximately $10 \mathrm{~m} \mathrm{~s}^{-1}$. However, the blowing air speeds at the end of the contraction section ranged from 4 to $24 \mathrm{~m} \mathrm{~s}^{-1}$ during tests. Consequently, isokinetic sampling might not be assured when extracting air samples. If the discharge air speed at the end of the contraction section was less than $10 \mathrm{~m} \mathrm{~s}^{-1}$, the sampled spore concentrations were lower than those in the wind tunnel. However, the maximum concentration error due to such superisokinetic sampling was $10 \%$ for the blowing air speed of $4 \mathrm{~m} \mathrm{~s}^{-1}$ in the wind tunnel (Rader and Marple, 1988). For contrast, if the blowing air speed was greater than $10 \mathrm{~m} \mathrm{~s}^{-1}$, the subisokinetic sampling was resulted and the sampled spore concentrations were higher than those in the wind tunnel. The maximum subisokinetic sampling error was approximately $20 \%$ at the blowing air speed of $24 \mathrm{~m} \mathrm{~s}^{-1}$.

In addition, some of the airborne spores may have settled on surfaces of the test section and would consequently have been omitted by the bioaerosol sensor. After each aerosolization test, the surfaces of the test section were sampled by the sterile cotton to quantify the settled spores. The results showed that the quantity of settled spores was much less than that of the collected spores by the sampler, and the losses on the surfaces were less than $0.1 \%$. Thus, the settled spores on the surfaces can be neglected in estimating the quantity of aerosolized spores from fungal colonies.

\section{Prediction of the Release Proportion for an Unknown Colony with a Homogeneous Age}

To predict the release proportion of an unknown colony grown at similar conditions, one can measure the respiratory intensity and identify the growth age according to Fig. 4, if the growth age is less than 14 days. The information in Fig. 6 can then be used to predict the spore release proportion. Note that in Figs. 4 and 6, both the respiratory intensities and the spore release proportions for the homogeneous colony were measured at an interval of two days in growth age. Linear interpolation could be used if the estimated growth age of an unknown colony did not fall at the measured points. To illustrate Steps 3 and 4 in the flow chart in Fig. 3, two types of colonies, with homogeneous (in the present section) and heterogeneous (in Section 3.4) age distributions, were employed as test samples.

An actual nine-day-old colony, as shown in Fig. 7(A), was used as an example homogeneous colony to validate the prediction of spore release proportion by the global method. 


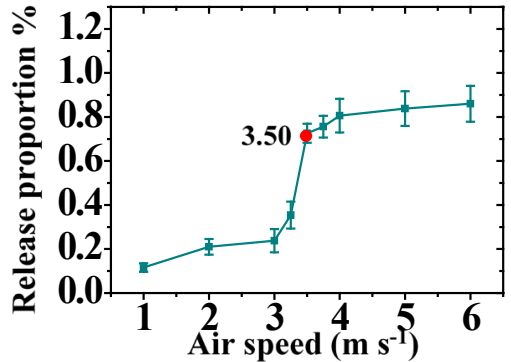

(A)

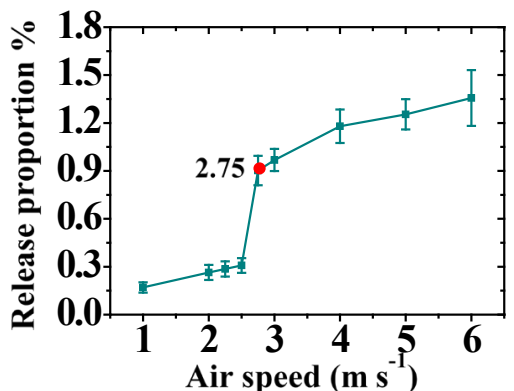

(D)

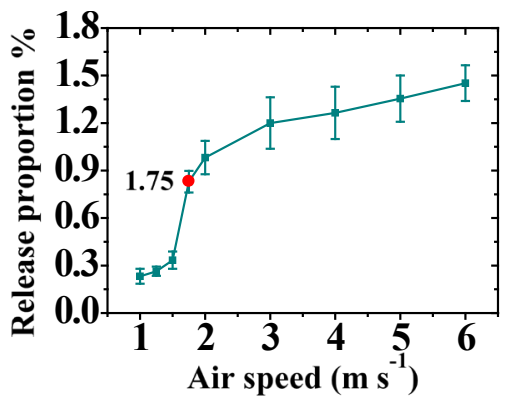

(G)

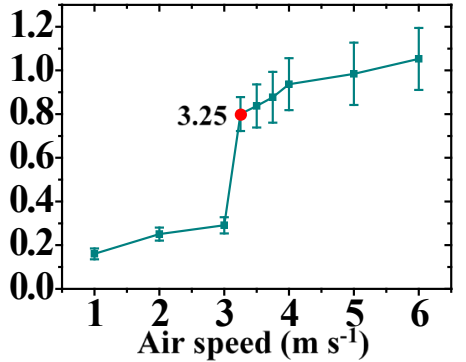

(B)

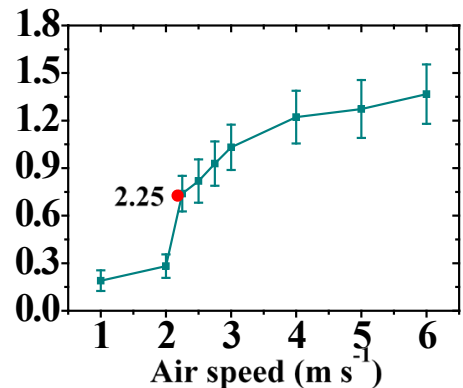

(E)

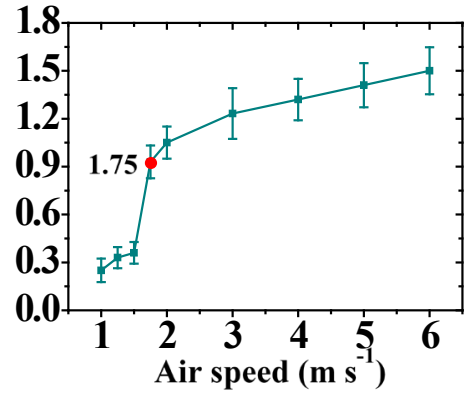

(H)

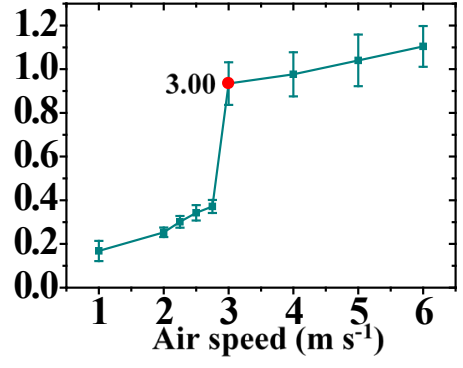

(C)

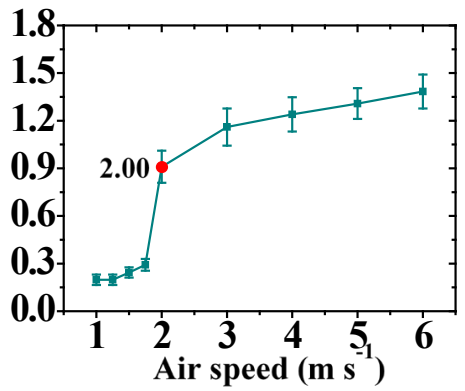

(F)

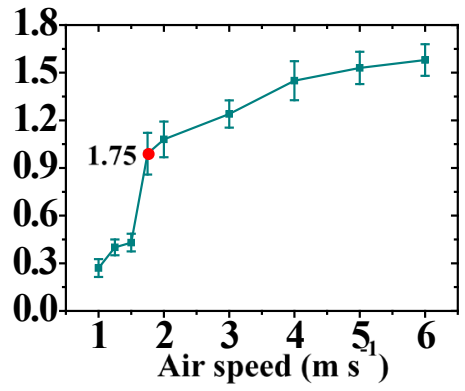

(I)

Fig. 6. Relationship between spore release proportion and blowing air speed for homogeneous colonies at different growth ages, where error bars represent the standard deviations for five repeated measurements: (A) four-day-old colony; (B) sixday-old colony; (C) eight-day-old colony; (D) 10-day-old colony; (E) 12-day-old colony; (F) 14-day-old colony; (G) 16day-old colony; (H) 18-day-old colony; (I) 20-day-old colony.

The release of carbon dioxide by the test colony was $84 \pm$ $6 \mu \mathrm{g} \mathrm{day}^{-1}$, and the total number of spores was $(1.16 \pm 0.09)$ $\times 10^{7}$. Hence, the respiratory intensity of the homogeneous colony was determined as $72 \pm 5 \mu \mathrm{g}\left(10^{7} \text { spores }\right)^{-1}$. The growth age of the colony was estimated as $9.1 \pm 0.6$ days on the basis of Fig. 4, which was very close to the actual growth age.

An age-weighted linear interpolation between Fig. 6(C) for an eight-day-old colony and Fig. 6(D) for a 10-day-old colony produced the spore release proportion for a growth age of 9.1 days. Fig. 7(B) compares the spore release proportion predicted by the global method with the actual release proportion obtained in the aerosolization tests, under different blowing air speeds. The relative errors of the spore release proportions between the prediction and the measurement were less than $10 \%$. The results demonstrated that the proposed method can predict the spore release proportion of a homogeneous colony with a growth age less than 14 days.

\section{Prediction of the Spore Release Proportion for Colonies with Heterogeneous Ages}

Two colonies with heterogeneous ages were employed as test samples. One had a maximum growth age of six days, as shown in Fig. 8(A), and the other had a maximum growth age of eight days, as shown in Fig. 8(B). The heterogeneous colonies grew from center to outer edge, and thus the sub colony in the center had the maximum growth age. Both the global prediction and partial prediction methods were used and compared.

Table 1 shows the respiratory intensities of the two heterogeneous colonies, where each colony was treated as a whole for measurement. For the maximum six-day-old colony, the respiratory intensity was $5875 \mu \mathrm{g}\left(10^{7} \text { spores }\right)^{-1}$, which corresponded to an average growth age of 2.9 days according to Fig. 4. Similarly, the global method determined the average age of the maximum eight-day-old colony as 4.1 days. Because the colonies grew from the center to the outward, the growth ages of the center sub-colonies were maximum, compared with the edge sub-colonies (Fig. S2). Thus, the average growth ages determined by the global method for both colonies were lower than their actual maximum growth ages in the center sub-colonies.

Fig. 9 compares the predicted release proportion of the 


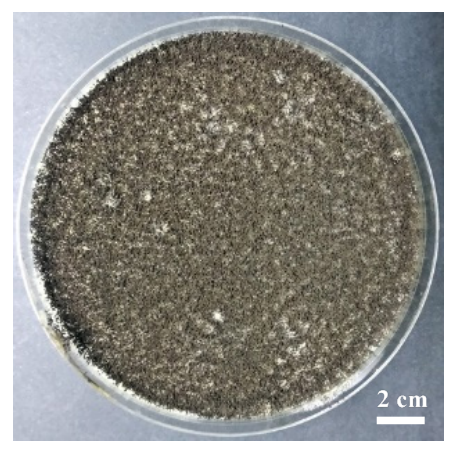

(A)

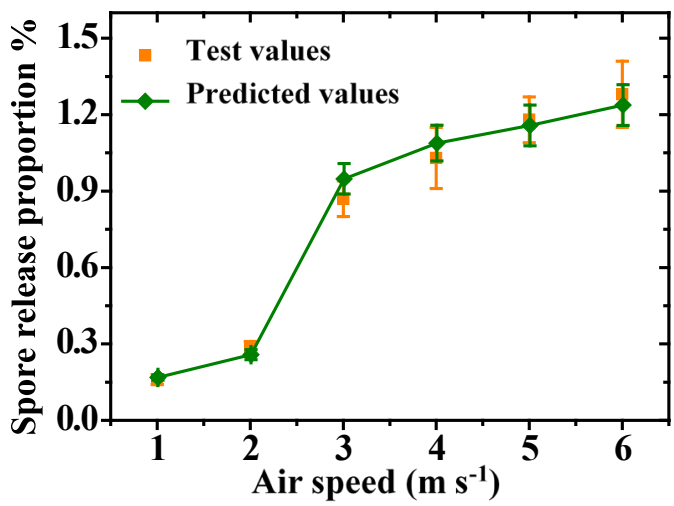

(B)

Fig. 7. Image of a homogeneous colony of $A$. niger and comparison of the predicted and measured spore release proportions for the colony: (A) the homogeneous colony with an actual growth age of nine days; (B) comparison of the spore release proportions predicted by the global method and obtained in measurements, where error bars represent the standard deviations for five repeated measurements.

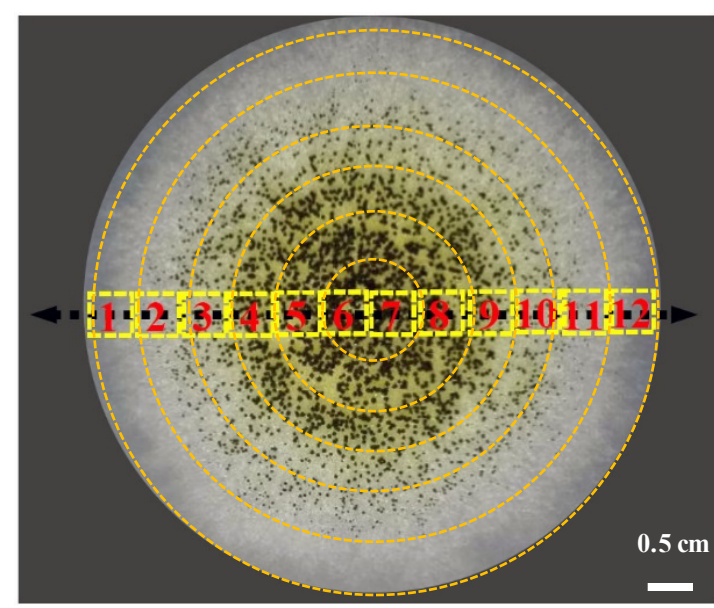

(A)

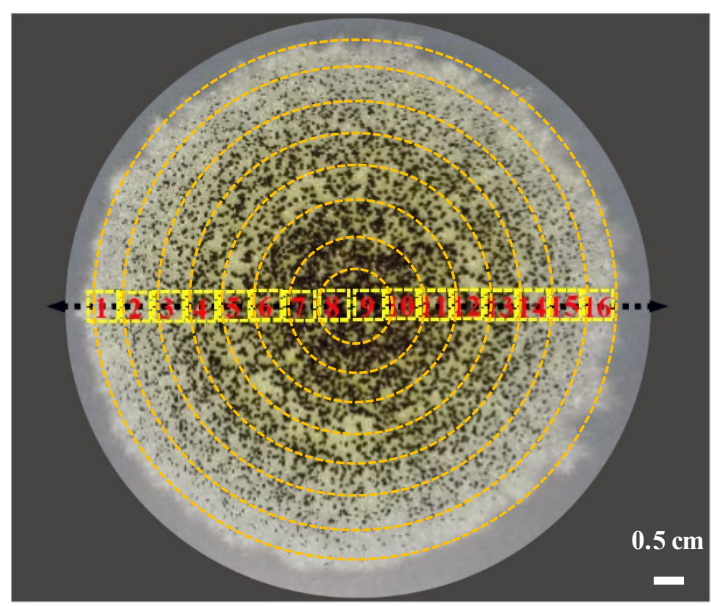

(B)

Fig. 8. Two colonies with heterogeneous ages and division of the colonies into square sub-colonies along the center line: (A) an actual maximum six-day-old colony; (B) an actual maximum eight-day-old colony.

Table 1. Respiratory intensity and average growth age of the heterogeneous colonies according to the global method.

\begin{tabular}{lll}
\hline Actual maximum growth age (day) & Respiratory intensity $\left(\mu \mathrm{g}\left(10^{7} \#\right)^{-1}\right)$ & Average age (day) \\
\hline 6 & $5875 \pm 123$ & $2.9 \pm 0.3$ \\
8 & $374 \pm 25$ & $4.1 \pm 0.4$ \\
\hline
\end{tabular}

two heterogeneous colonies by the global method with the actual measured values. The global method provided the spore release proportions based on the age-weighted linear interpolation of Fig. 4, and the results were in general agreement with the measured values. However, the global prediction determined smaller release proportions when the blowing air speed was greater than $3.00 \mathrm{~m} \mathrm{~s}^{-1}$. The maximum relative error in the predicted release proportions as compared with the test values was $43 \%$. Thus, the global method can be utilized only for a rough estimation of the spore release proportion with a heterogeneous age.

In contrast, the partition prediction method divided the heterogeneous colony into many annuli, as shown in Fig. 8.
The representative square sub-colonies in each annulus along the center line were analyzed. The dimensions of each sub-colony were $0.5 \mathrm{~cm} \times 0.5 \mathrm{~cm}$. Because the diameters of the maximum six-day-old and eight-day-old colonies were approximately 6 and $8 \mathrm{~cm}$, respectively, the two colonies were divided into 12 and 16 sub-colonies. The respiratory intensity of each individual sub-colony and the corresponding release proportion were determined.

Fig. 10 presents the respiratory intensities of different square sub-colonies for the two heterogeneous colonies. The respiratory intensity profiles were almost parabolic, and the respiratory intensity was lowest in the center. For the maximum six-day-old colony (Fig. 10(A)), sub-colonies one 


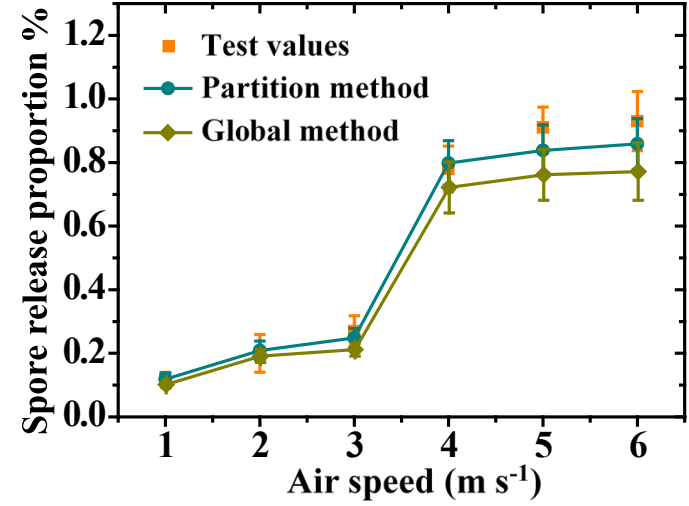

(A)

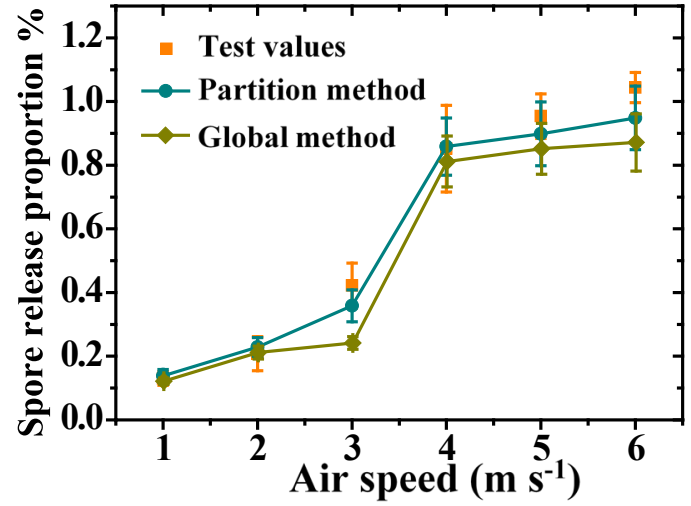

(B)

Fig. 9. Comparison of the spore release proportion for the heterogeneous colonies predicted by two methods with the test values, where error bars represent the standard deviations for five repeated measurements: (A) an actual maximum six-dayold colony; (B) an actual maximum eight-day-old colony.
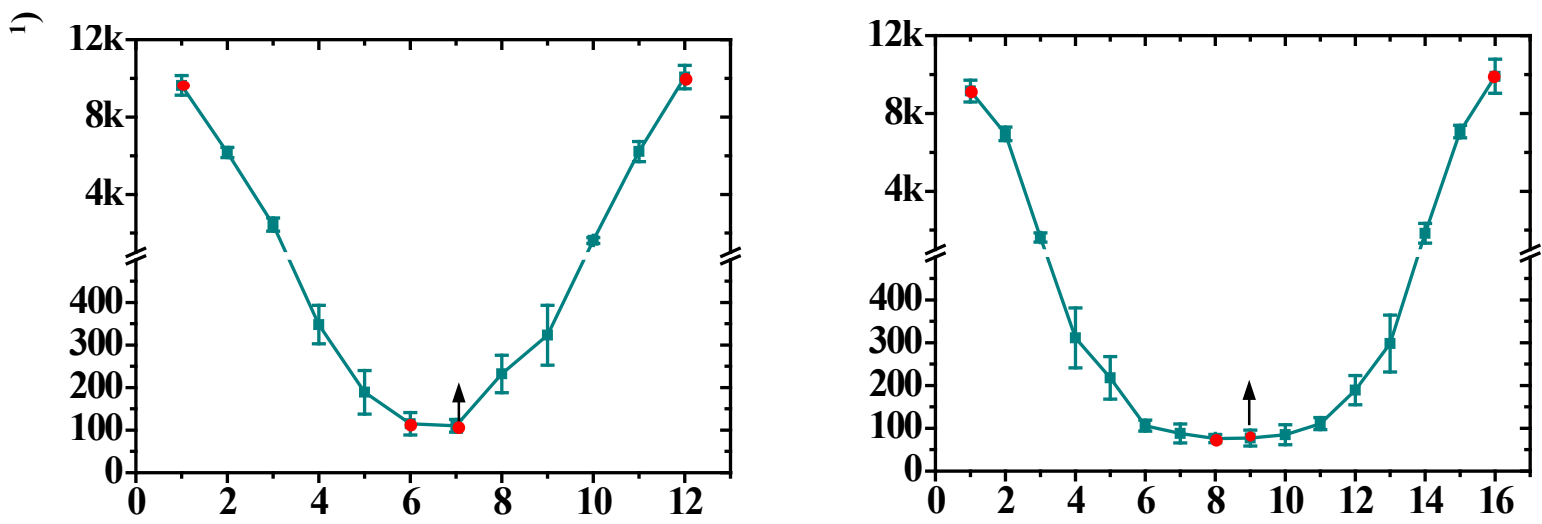

Fig. 10. Variation in the respiratory intensity of the square sub-colony with the sub-colony sequence number, where error bars represent the standard deviations for five repeated measurements: (A) an actual maximum six-day-old colony; (B) an actual maximum eight-day-old colony.

and 12 had the maximal respiratory intensities of 9,635 and $10,063 \mu \mathrm{g}\left(10^{7}\right.$ spores $^{-1}$, respectively. Sub-colonies six and seven had the minimal respiratory intensities of 115 and $110 \mu \mathrm{g}\left(10^{7} \text { spores }\right)^{-1}$, respectively. The growth ages of the above sub-colonies are indicated by red dots in the figure. The growth ages of sub-colonies one and 12 were approximately 1.8 and 1.7 days, respectively, and they were the youngest among the twelve sub-colonies. Sub-colonies six and seven were oldest, with growth ages of 6.1 and 6.3 days, respectively. These two growth ages were close to the actual maximum growth age of six days. Similar results were obtained for the maximum eight-day-old colony, as shown in Fig. 10(B).

Because the original spores were inoculated at the center of the nutrient agar, the mycelia grew from the center to outer edge and formed the circular colony with heterogeneous ages of spores. The growth ages of the sub-colonies in the same annulus were similar, as shown in Fig. 8. The released spores in the annulus sub-colonies were estimated from the average release proportion of the two representative square sub-colonies in the annulus multiplied by the total grown spores therein. The release proportion for the whole colony was calculated by Eq. (1) for each annulus with divisions as shown in Fig. 8.

Fig. 9 compares the predicted release proportion of the two heterogeneous colonies by the partition method with the actual measured values. The partition method provided more accurate spore release proportions than the global method, especially when the blowing air speed was greater than $3.00 \mathrm{~m} \mathrm{~s}^{-1}$. The relative errors of the predicted spore release proportions by the partition method as compared with the test values were all less than $10 \%$. Thus, the partition method is more accurate in predicting the spore release proportion with a heterogeneous age.

For the colonies with heterogeneous ages, the predicted spore release proportion for a maximum eight-day-old colony under a blowing speed of $3.00 \mathrm{~m} \mathrm{~s}^{-1}$ by the global method was significantly lower than that predicted by the partition method (Fig. 9(B)). The average growth age of the whole colony was estimated as 4.1 days by the global method, which is very close to four days. The threshold air speed for 
significant detachment of spores from the four-day-old colony was $3.50 \mathrm{~m} \mathrm{~s}^{-1}$ (Fig. 6(A)), which is higher than the blowing speed of $3.00 \mathrm{~m} \mathrm{~s}^{-1}$. Thus, the predicted spore release proportion for the actual blowing speed of $3.00 \mathrm{~m} \mathrm{~s}^{-1}$ was underestimated. However, according to the partition method, the growth ages of sub-colonies four through eight were greater than 4.1 days. Consequently, the spore release proportions for these sub-colonies as estimated by the partition method were much higher than those estimated by the global prediction method. The averaging of the growth age of the heterogeneous colony by the global method resulted in underestimation of the spore release proportions, especially under a blowing air speed higher than $3.00 \mathrm{~m} \mathrm{~s}^{-1}$.

The results indicate that respiratory intensity can be used to predict the spore aerosolization of $A$. niger colonies. Although some previous studies have conducted experimental tests to determine the release potential of fungal spores, the predicted spore release rate was inaccurate (Gorny et al., 2001; Kesavan et al., 2019; Wang et al., 2020). One reason is that the growth age has a significant effect on spore detachment (Sivasubramani et al., 2004; Frankel et al., 2014). The close correlation between respiratory intensity and growth age was revealed in this study.

\section{Effects of Environmental Factors and Fungal Species on the Respiratory Intensity and Spore Aerosolization}

Environmental factors, such as relative humidity, temperature, nutrition content of the nutrient agar and oxygen content in the jar, can affect the respiratory intensity of colonies (Willcock and Magan, 2000). In this study, all of the respiratory intensities were measured in the standard conditions, though the measured respiratory intensity might not be identical to those of the colonies growing in actual indoor environments. In addition, the relative humidity and temperature may also affect the aerosolization of spores. Spores were found to be more easily aerosolized in dry environments (Frankel et al., 2014). Temperature might affect the binding force between spores and conidiophores of fungal colonies. Because this study focused on the prediction method itself, the realistic environmental factors were not accounted for. For estimating the spore aerosolization from general building materials, some additional works may be required. A researcher may have to establish relationship of the spore release rate versus age of the fungi on the specific materials. Nevertheless, one may use the results in this work for a rough estimation if lacking of the aforementioned precise relationship, because the spore release potential is mainly subject to their growth age.

This investigation adopted the pure $A$. niger species for the experimental tests. The results of respiratory intensity and aerosolization tests for $A$. niger may not be applicable for other fungi (Ryan and Law, 2005). In the future, other common fungi in indoor environments should be further investigated.

\section{CONCLUSIONS}

This paper proposed the use of respiratory intensity as an indicator of growth age and also for prediction of the spore aerosolization of Aspergillus niger colonies. Both the global method, which treats the colony as a whole, and the partition method, which divides the colony into many sub-colonies, were adopted to predict the spore release proportion. Two types of colonies, with homogeneous and heterogeneous age distributions, were aerosolized in a wind tunnel for determination of the spore release proportion and validation of the method. It was found that the respiratory intensity of the homogeneous colony correlated well with the growth age when the age was less than 14 days under standard growth conditions. The two-day-old colony had a maximal respiratory intensity of $8,781 \mu \mathrm{g}\left(10^{7} \text { spores }\right)^{-1}$, while the 14 day-old colony had a minimum respiratory intensity of $47 \mu \mathrm{g}\left(10^{7} \text { spores }\right)^{-1}$. The spore release proportion increased with the blowing air speed and the growth age of the colony, while the threshold air speed for significant detachment of spores decreased with an increase in growth age. When the blowing speed was $3.00 \mathrm{~m} \mathrm{~s}^{-1}$, the release proportion for the 14-day-old colony $(1.16 \%)$ was 4.0 times that for the sixday-old colony $(0.29 \%)$. The spore release proportion for the colony with heterogeneous ages as predicted by the partition method was more precise than that predicted by the global method. The relative errors of the predicted spore release proportions by the partition method as compared with the test values were less than $10 \%$. The averaging of the growth age of the heterogeneous colony by the global method resulted in underestimation of the spore release proportions, especially under a blowing air speed higher than $3.00 \mathrm{~m} \mathrm{~s}^{-1}$.

\section{ACKNOWLEDGMENTS}

This work was supported by the National Natural Science Foundation of China (Grant No.: 51622804 and 51978450).

\section{SUPPLEMENTARY MATERIAL}

Supplementary data associated with this article can be found in the online version at http://www.aaqr.org.

\section{REFERENCES}

Atkin, O., Millar, H. and Turnbull, M. (2010). Plant respiration in a changing world. New Phytol. 187: 269-272.

Fang, Z., Zhang, J., Guo, W. and Lou, X. (2019). Assemblages of culturable airborne fungi in a typical urban, tourism-driven center of southeast China. Aerosol Air Qual. Res. 19: 820-831.

Frankel, M., Hansen, E.W. and Madsen, A.M. (2014). Effect of relative humidity on the aerosolization and total inflammatory potential of fungal particles from dustinoculated gypsum boards. Indoor Air 24: 16-28.

Gervais, P., Abadie, C. and Molin, P. (1999). Fungal turgor pressure is directly involved in the hyphal growth rate. Microbiol. Res. 154: 81-87.

Gopalakrishnan, S., Devassikutty, A.K., Mathew, M., Ayyappan, D., Thiagarajan, S. and Raghunathan, R. (2016). Passive release of fungal spores from synthetic solid waste surfaces. Aerosol Air Qual. Res. 16: 1441-1451.

Gorny, R.L., Reponen, T., Grinshpun, S.A. and Willeke, K. 
(2001). Source strength of fungal spore aerosolization from moldy building material. Atmos. Environ. 35: 4853-4862.

Hugenholtz, P. and Fuerst, J.A. (1992). Heterotrophic bacteria in an air-handling system. Appl. Environ. Microbiol. 58: 3914-3920.

Humbal, C., Gautam, S. and Trivedi, U. (2018). A review on recent progress in observations, and health effects of bioaerosols. Environ. Int. 118: 189-193.

Kallawicha, K., Chuang, Y.C., Lung, S.C.C., Wu, C.F., Han, B.C., Ting, Y.F. and Chao, H.J. (2018). Outpatient visits for allergic diseases are associated with exposure to ambient fungal spores in the greater Taipei area. Aerosol Air Qual. Res. 18: 2077-2085.

Kesavan, J., Kilper, G., Williamson, M., Alstadt, V., Dimmock, A. and Bascom, R. (2019). Laboratory validation and initial field testing of an unobtrusive bioaerosol detector for health care settings. Aerosol Air Qual. Res. 19:331-344.

Kildeso, J., Wurtz, H., Nielsen, K.F., Kruse, P., Wilkins, K., Thrane, U., Gravesen, S., Nielsen, P.A. and Schneider, T. (2003). Determination of fungal spore release from wet building materials. Indoor Air 13: 148-155.

Krijgsheld, P., Bleichrodt, R.V., Van Veluw, G.J., Wang, F., Müller, W.H., Dijksterhuis, J. and Wösten, H.A.B. (2013). Development in Aspergillus. Stud. Mycol. 74: 1-29.

Li, X., Zhang, T.F. and Wang, S.G. (2018). Measuring detachment of Aspergillus niger spores from colonies with an atomic force microscope. Indoor Air 28: 744-753.

Li, X., Zhang, T.F., Wang, J. and Wang, S.G (2019). Aerosolization of Aspergillus niger spores from growing colonies on a bare tube. Atmos. Environ. 218: 117008.

Liu, T., Chen, L.W.A., Zhang, M., Watson, J.G., Chow, J.C., Cao, J., Chen H., Wang W., Zhang J., Zhan, C., Liu, H., Zheng, J., Chen, N., Yao, R. and Xiao, W. (2019). Bioaerosol concentrations and size distributions during the autumn and winter seasons in an industrial city of central China. Aerosol Air Qual. Res. 19: 1095-1104.

Meharzi, S., Mansouri, R., Chekchaki, N., Bouchair, N., Belgharssa, A., Tridon, A., Messarah, M. and Boumendjel, A. (2017). Indoor aeroallergens in asthmatic pediatric population in annaba (Algeria). Aerosol Air Qual. Res. 17: 2482-2490.

Mensah-Attipoe, J., Saari, S., Veijalainen, A.-M., Pasanen, P., Keskinen, J., Leskinen, J.T.T. and Reponen, T. (2016). Release and characteristics of fungal fragments in various conditions. Sci. Total Environ. 547: 234-243.

Milton, D.K., Fabian, M.P., Cowling, B.J., Grantham, M.L. and McDevitt, J.J. (2013). Influenza virus aerosols in human exhaled breath: Particle size, culturability, and effect of surgical masks. PLoS Pathog. 9: e1003205.

Mu, F., Li, Y., Lu, R., Qi, Y., Xie, W. and Bai, W. (2019). Source identification of airborne bacteria in the mountainous area and the urban areas. Atmos. Res. 231: 104676.

Norback, D., Lu, C., Zhang, Y., Li, B., Zhao, Z., Huang, C., Zhang, X., Qian, H., Sun, Y., Sundell, J., Juan, W., Liu, W. and Deng, Q. (2019). Onset and remission of childhood wheeze and rhinitis across China - Associations with early life indoor and outdoor air pollution. Environ. Int. 123: 61-69.

Park, J.H., Sulyok, M., Lemons, A.R., Green, B.J. and Cox-
Ganser, J.M. (2018). Characterization of fungi in office dust: Comparing results of microbial secondary metabolites, fungal internal transcribed spacer region sequencing, viable culture and other microbial indices. Indoor Air 28: 708-720.

Piekarska, K., Trusz, A. and Szczesniak, S. (2018). Bacteria and fungi in two air handling units with air recirculating module. Energy Build. 178: 154-164.

Qi, Z.T., Kong, H.Z. and Sun, Z.M. (1997). Flora Fungorum Sinicorum. Science Press, Beijing (in Chinese).

Rader, D.J. and Marple, V.A. (1988). A study of the effects of anisokinetic sampling. Aerosol Sci. Technol. 8: 283299.

Rim, D. and Novoselac, A. (2009). Transport of particulate and gaseous pollutants in the vicinity of a human body. Build. Environ. 44: 1840-1849.

Ryan, M.G. and Law, B.E. (2005). Interpreting, measuring, and modeling soil respiration. Biogeochemistry 73: 3-27.

Sivasubramani, S.K., Niemeier, R.T., Reponen, T. and Grinshpun, S.A. (2004). Assessment of the aerosolization potential for fungal spores in moldy homes. Indoor Air 14: 405-412.

Teertstra, W.R., Tegelaar, M., Dijksterhuis, J., Golovina, E.A., Ohm, R.A. and Wosten, H.A.B. (2017). Maturation of conidia on conidiophores of Aspergillus niger. Fungal Genet. Biol. 98: 61-70.

Wang, P., Yuan, S., Yang, N., Wang, A., Fordjour, A. and Chen, S. (2020). The collection method for crop fungal spores based on an efficient microfluidic device. Aerosol Air Qual. Res. 20: 72-79.

Wang, Y., Fu, Y., Wang, C. and Wen, N. (2018). Dissimilar emission characteristics between bioaerosol and suspended particles from gaseous biofilters and bioaerosol health risk evaluation. Aerosol Air Qual. Res. 18: 1874-1885.

Wei, J., Li, Z., Peng, Y. and Sun, L., (2019). MODIS Collection 6.1 aerosol optical depth products over land and ocean: Validation and comparison. Atmos. Environ. 201: 428-440.

Willcock, J. and Magan, N., (2000). Impact of environmental factors on fungal respiration and dry matter losses in wheat straw. J. Stored Prod. Res. 37: 35-45.

Wu, Y., Chen, A.L., Luhung, I., Gall, E.T., Cao, Q.L., Chang, V.W.C. and Nazaroff, W.W. (2016). Bioaerosol deposition on an air-conditioning cooling coil. Atmos. Environ. 144: 257-265.

Yao, M. (2018). Bioaerosol: A bridge and opportunity for many scientific research fields. J. Aerosol Sci. 115: 108-112.

Zhang, S., Du, R., Chen, H., Ren, W. and Du, P. (2019). Seasonal variation of microbial activity and pathogenic bacteria under non-serious pollution levels in Beijing. Aerosol Air Qual. Res. 19: 1798-1807.

Zhao, P.C., Chan, P.O., Gao, Y.S., Lai, O.W., Zhang, T. and Li, Y.G. (2019). Physical factors that affect microbial transfer during surface touch. Build. Environ. 158: 28-38.

Received for review, January 16, 2020 Revised, March 21, 2020 Accepted, March 21, 2020 